\title{
A NOTE ON THE IDEMPOTENT MEASURES ON COUNTABLE SEMIGROUPS
}

\author{
TZE-CHIEN SUN and N. A. TSERPES
}

(Received 1 July 1969)

Communicated by B. Mond

In [6] we announced the following

CONJECTURE: Let $S$ be a locally compact semigroup and let $\mu$ be an idempotent regular probability measure on $S$ with support $F$. Then

(a) $F$ is a closed completely simple subsemigroup.

(b) $F$ is isomorphic both algebraically and topologically to a paragroup ([2], p. 46) $X \times G \times Y$ where $X$ and $Y$ are locally compact left-zero and right-zero semigroups respectively and $G$ is a compact group. In $X \times G \times Y$ the topology is the product topology and the multiplication of any two elements is defined by

$$
(x, g, y)\left(x^{\prime}, g^{\prime}, y^{\prime}\right)=\left(x, g\left[y, x^{\prime}\right] g^{\prime}, y^{\prime}\right),
$$

where $\left[y, x^{\prime}\right]$ is continuous mapping from $Y \times X \rightarrow G$.

(c) The induced $\mu$ on $X \times G \times Y$ can be decomposed as a product measure $\mu_{X} \times \mu_{G} \times \mu_{Y}$ where $\mu_{X}$ and $\mu_{Y}$ are two regular probability measures on $X$ and $Y$ respectively and $\mu_{G}$ is the normed Haar measure on $G$.

It was shown in the Theorem 1 of [6] that if (a) holds, then (b) and (c) always follow. Hence, the crucial part of the conjecture is actually part (a).

For the case $S$ is compact, the conjecture was already proved in [3] and [5]. For discrete $S$, it was proved in [4]. When $S$ is abelian, any completely simple subsemigroup $S$ reduces to a group. We showed in [8] that in this case $F$ is a compact group and $\mu$ is the normed Haar measure on $F$. In this note we investigate the case when $S$ is countable but not necessarily discrete, in order to gain some insight to the above conjecture. It turns out that our conjecture is true for any countable Hausdorff $S$, even without the assumption of local compactness. The result in this note and that in [7], where $S$ is assumed to be completely regular and completely simple itself, seem to indicate that the assumption of local compactness is not essential to the conjecture. This assumption only makes the integration on $S$ easier, owing to the well established integration theory on locally compact spaces.

Theorem. Let $S$ be a countable Hausdorff semigroup and let $\mu$ be an idempotent regular probability measure on $S$ with support $F$. Let $P=\{s \in F ; \mu[s]>0\}$. 
Then $\bar{P}=F$ and $P$ is a completely simple subsemigroup. $(\bar{P}=$ the closure of $P$ in $S$ ). Moreover $F$ is a closed completely simple subsemigroup and $F=X \times G \times Y$, where $X$ and $Y$ are left-zero and right-zero countable semigroups respectively and $G$ is a finite group. Moreover $\mu=\mu_{X} \times \mu_{G} \times \mu_{Y}$ as in the conjecture.

Proof. (i) That $\bar{P}=F$ is clear.

(ii) $P$ is a semigroup because if $u, v \in P$ then

$$
\mu(u v) \geqq \mu\left(u v v^{-1}\right) \mu(v) \geqq \mu(u) \mu(v)>0
$$

by idempotence. Also, from the assumption of idempotence, it is easy to see that either $P=\{0\}$ or $P$ contains no zero element. We shall therefore consider only the latter case.

(iii) For any $z \in P$

$$
\begin{aligned}
\mu(P z) & =\sum_{x \in P z} \mu\left(P z x^{-1}\right) \mu(x)+\sum_{x \in P-P z} \mu\left(P z x^{-1}\right) \mu(x) \\
& =\mu(P z)+\sum_{x \in P-P z} \mu\left(P z x^{-1}\right) \mu(x)
\end{aligned}
$$

since $\mu\left(P z x^{-1}\right)=1$ for $x \in P z$. It follows $\mu\left(P z x^{-1}\right)=0$ for all $x \in P-P z$ so $P-P z$ is a left ideal of $P$, and $z \in P z$ because $\left(P z z^{-1}\right)=1$. Similarly, $z \in z P$. Let $z=z k$ for some $k \in P$. Then $k \notin P-P z$ because otherwise $z=z k \in P-P z$ which is a contradiction. Therefore, we have for any $z \in P, z=z p z$ for some $p \in P$ and $P$ is regular [1].

Let $e$ be an idempotent in $P$ and suppose that $e f=f e=f$ for some other idempotent $f$ in $P$. Then $e \in P f$, for otherwise $f e=f$ is in $P-P f$, a contradiction. It follows that $e f=e=f$ and that every idempotent in $P$ is primitive. Hence $P$ is completely simple ([1], p. 84).

(iv) Let $e$ be any idempotent in $P$. Let $X^{\prime}$ and $Y^{\prime}$ be the sets of idempotents in $P e$ and $e P$ respectively. Let $G=e P e$. Then $X^{\prime}$ and $Y^{\prime}$ are left-zero and right-zero semigroups resp. and $G$ is a group. $P$ is algebraically isomorphic to $X^{\prime} \times G \times Y^{\prime}$ with the multiplication given in (1) with $\left[y, x^{\prime}\right]=y x^{\prime}$. The isomorphism is given by ([2], p. 47-48)

$$
\begin{gathered}
\eta: X^{\prime} \times G \times Y^{\prime} \rightarrow P, \eta\left(x^{\prime}, g, y^{\prime}\right)=x^{\prime} g y^{\prime} \\
\eta^{-1}: P \rightarrow X^{\prime} \times G \times Y^{\prime}, \eta^{-1}(s)=\left(s(e s e)^{-1}, e s e,(e s e)^{-1} s\right) .
\end{gathered}
$$

(v) For any $a \in Y^{\prime}$, write $S_{a}=\left\{\left(x^{\prime}, g, y^{\prime}\right) \in P ; y^{\prime}=a\right\}$. For any $s \in S_{a}$ the measure $\mu_{s}(\cdot)=\mu\left(\cdot s^{-1}\right)$ has the following property ([3], p. 181), for any $B \in S_{a}$,

$$
\mu_{s}(B)=\mu\left(B s^{-1}\right)=\sum_{y \in S_{a}} \mu_{y}(B) \mu_{s}\{y\} .
$$

$B \subset S_{a}$ is called invariant set if $\mu_{s}(B)=1$ for all $s \in B$. It follows that if $B$ is invariant, then $P B \subset B$ and since $S_{a}$ is left simple (in fact left group), $B=S_{a}$. Note that since $S$ is countable, every subset is countable and measurable and integration 
reduces to summation in this case. By the method developed in ([3], p. 181-183), we have, for any $B \subset S_{a}, \mu\left(B s^{-1}\right)=$ constant independent of $s \in S_{a}$. Since $G \subset S_{e}$, where $e$ is the unit of $G$, we have for $c \in G$

$$
\mu(c) \geqq \sum_{b \in G} \mu(b) \mu\left(c b^{-1}\right)=\sum_{b \in G} \mu(b) \mu\left(c c^{-1}\right) \geqq \mu(G) \mu(e)>0
$$

since $\mu\left(c b^{-1}\right)=\mu\left(c c^{-1}\right) \geqq \mu(e)$. Hence $G$ must be a finite group. It follows that inversion in $G$ is continuous. Therefore the mapping $\eta$ and $\eta^{-1}$ defined in (2) are continuous and $P$ is also homeomorphic to $X^{\prime} \times G \times Y^{\prime}$.

(vi) Let $X$ and $Y$ be the closures of $X^{\prime}$ and $Y^{\prime}$ in $F$. Then $X$ and $Y$ are again left-zero and right-zero semigroups resp., $X \cap Y=X^{\prime} \cap Y^{\prime}=\{e\}$ and since $G$ is closed, $X \times G \times Y$ is again a completely simple semigroup with $[y, x]=y x$ in (1). We can extend $\eta$ to $X \times G \times Y \rightarrow F$ as follows. For any $(x, g, y) \in X \times G \times Y$, there exist $\left(x_{\alpha}, g_{\alpha}, y_{\alpha}\right) \rightarrow(x, g, y),\left(x_{\alpha}, g_{\alpha}, y_{\alpha}\right) \in X^{\prime} \times G \times Y^{\prime}$. Then $\eta\left(x_{\alpha}, g_{\alpha}, y_{\alpha}\right)=$ $x_{\alpha} g_{\alpha} y_{\alpha} \rightarrow x g y \in F$. We define $\bar{\eta}(x, g, y)=x g y=s \in F$. Using the continuity of multiplication in $F$ it is not hard to show that $\bar{\eta}$ is a homomorphism. To show that $\bar{\eta}$ is onto, let $s \in F$. There is $\left\{s_{\alpha}\right\} \in P$, such that $s_{\alpha} \rightarrow s$. Then

and

$$
\eta\left(s_{\alpha}\left(e s_{\alpha} e\right)^{-1}, e s_{\alpha} e,\left(e s_{\alpha} e\right)^{-1} s_{\alpha}\right)=s_{\alpha} \rightarrow s,
$$

$$
\left(s_{\alpha}\left(e s_{\alpha} e\right)^{-1}, e s_{\alpha} e,\left(e s_{\alpha} e\right)^{-1} s_{\alpha}\right) \rightarrow\left(s(e s e)^{-1}, e s e,(e s e)^{-1} s\right)
$$

so that $\bar{\eta}\left(s(e s e)^{-1}\right.$, ese, $\left.(e s e)^{-1} s\right)=s$. It is also easy to see that $\bar{\eta}$ is one-one. So $\bar{\eta}$ is an algebraic isomorphism. Hence $F$ is completely simple (algebraically). But $\bar{\eta}$ and $\bar{\eta}^{-1}$ are continuous because $G$ is finite and $g \rightarrow g^{-1}$ is continuous in $G$ and multiplication on $F$ is jointly continuous. Hence $F=X \times G \times Y$.

(vii) The fact that $\mu$ can be decomposed as a product measure $\mu_{X} \times \mu_{G} \times \mu_{Y}$ on $X \times G \times Y$ can be proved by a method similar to one used in [3] (pp. 183-184), using the fact that $\mu\left(B s^{-1}\right)=$ constant for all $s \in S_{a}$, for some $a \in Y$.

Remark. Part (iii) can be proved by a method given in [4](p. 90) by considering $P$ as discrete semigroup and using some known results in the theory of Markov chains. However our proof here is new and non-probabilistic.

\section{References}

[1] A. H. Clifford and G. B. Preston, The algebraic theory of semigroups, Vol. 1, (Mathematical Surveys, No. 7, American Math. Society, 1961).

[2] J. F. Berglund and K. H. Hofmann, Compact semitopological semigroups and weakly periodic functions (Springer Lecture Notes No. 42, 1967).

[3] M. Heble and M. Rosenblatt, 'Idempotent measures on a compact topological semigroup', Proc. Amer. Math. Soc. 14 (1963), 177-184.

[4] P. Martin-Lof, 'Probability theory on discrete semigroups', Z. Wahrscheinlichkeitstheorie verw. Geb. 4 (1966), 78-102.

[5] J. S. Pym, 'Idempotent measures on semigroups', Pacific J. of Math. 12 (1962), 685-698. 
[6] Tze-Chien Sun and N. A. Tserpes, 'Idempotent measures on locally compact semigroups', $Z$. Wahrscheinlichkeitstheorie verw. Geb. (to appear).

[7] A. Tortrat, 'Lois tendues sur un demi-groupe topologique complètement simple, Z. Wahrscheinlichkeitstheorie verw. Geb. 6 (1966), 145-160.

[8] N. A. Tserpes and Tze-Chien Sun, 'Idempotent probability measures on locally compact abelian semigroups', Jour. Math. Mech. (to appear, July, 1970).

\author{
Wayne State University \\ and \\ University of South Florida
}

